PROCEEDINGS OF THE

AMERICAN MATHEMATICAL SOCIETY

Volume 132, Number 7 , Pages 2057-2066

S 0002-9939(04)07284-3

Article electronically published on January 20, 2004

\title{
BOUNDEDNESS OF SOLUTIONS FOR SEMILINEAR REVERSIBLE SYSTEMS
}

\author{
XIONG LI \\ (Communicated by Carmen C. Chicone)
}

\begin{abstract}
In this paper we will study the boundedness of all solutions for second-order differential equations

$$
\ddot{x}+f(x) \dot{x}+\lambda^{2} x+g(x)=p(t),
$$

where $\lambda \in R$ and $g(x)$ satisfies the sublinear growth condition. Since the system in general is non-Hamiltonian, we have to introduce reversibility assumptions to apply the twist theorem for reversible mappings. Under some suitable conditions we then obtain the existence of invariant tori and thus the boundedness of all solutions.
\end{abstract}

\section{INTRODUCTION}

We investigate the nonlinear oscillator

$$
\ddot{x}+f(x) \dot{x}+\lambda^{2} x+g(x)=p(t),
$$

where $\lambda \in R$ and $g(x)$ satisfies the sublinear growth condition, and we ask under what assumptions all solutions of (1.1) will be bounded in the $(x, \dot{x})$-phase plane for a $2 \pi$-periodic forcing $p$.

Before stating our result, we introduce some related results. First we consider the following conservative system:

$$
\ddot{x}+V_{x}(x, t)=0 .
$$

The first contribution of the boundedness of all solutions of (1.2) is due to Morris [16], who proved that all solutions of

$$
\ddot{x}+2 x^{3}=p(t)
$$

are bounded where $p(t) \in C^{0}\left(S^{1}\right)$. In 1987, Dieckerhoff and Zehnder [1] proved that all solutions of

$$
\ddot{x}+x^{2 n+1}+\sum_{i=0}^{2 n} p_{i}(t) x^{i}=0
$$

Received by the editors November 5, 2002 and, in revised form, March 2, 2003 and March 21, 2003.

2000 Mathematics Subject Classification. Primary 34C11.

Key words and phrases. Boundedness of solutions, KAM theory, reversible systems.

This project (10301006) was supported by the NSFC.

(C)2004 American Mathematical Society 
are bounded where $p_{i}(t) \in C^{\infty}\left(S^{1}\right)(i=0,1, \ldots, 2 n)$. Subsequently, this result was extended to more general cases for a large class of superlinear functions $V_{x}(x, t)$ in (1.2) by several authors; we refer to [5], [21], [23] and references therein.

On the other hand, the sublinear and semilinear cases are relatively new fields; see [4], 9], 10], 11], [12], [13], [17], [18, 19] and the references therein for details.

Next, we consider the following non-conservative system:

$$
\ddot{x}+f(x, t) \dot{x}+g(x, t)=0 .
$$

If the functions $f(x, t), g(x, t)$ in the equation (1.5) have certain symmetry, then (1.5) becomes a reversible system. Using the KAM theory for reversible systems 20], one can prove the existence of invariant tori and thus boundedness of all solutions. In 1991, Liu 15 proved that all solutions of

$$
\ddot{x}+\left(\sum_{i=0}^{m} a_{i} x^{2 i+1}\right) \cdot \dot{x}+x^{2 n+1}+\sum_{j=0}^{n-1} b_{j} x^{2 j+1}=p(t)
$$

are bounded where $n \geq 2(m+1)$ and $p(t)$ is odd and periodic in $t \in R^{1}$. Since then, several authors [7, [22, improved this result for the superlinear function $g(x, t)$. If the function $g(x, t)$ satisfies the sublinear growth condition and some other assumptions, the author [6] obtained the boundedness of all solutions of (1.5).

Recently, M. Kunze, T. Kupper and B. Liu [3] obtain some sharp results concerning the boundedness of solutions for semilinear reversible oscillators at resonance with the following form:

$$
\ddot{x}+f(x) \dot{x}+n^{2} x+\phi(x)=p(t)
$$

with $n \in N$ and $\phi(x)$ bounded. Another semilinear reversible system,

$$
\ddot{x}+f(x) \dot{x}+\omega^{2} x+\phi(x)=p(t),
$$

was considered in [8], where $\omega \notin N$ and $\phi(x)$ is bounded.

In this paper we shall study the boundedness of all solutions of (1.1) if $\lambda \in R$ and $g(x)$ satisfies the sublinear growth condition. In what follows, we denote by $G(x)$ and $F(x)$ the integrals of $g(x)$ and $f(x)$ with $G(0)=0$ and $F(0)=0$, respectively. We also denote by $c<1$ and $C>1$, respectively, two universal positive constants not concerning their quantities.

Suppose that:

(A1) $f, g, p \in C^{\infty}(R)$.

(A2) $f, g$ and $p$ are odd functions, and $p(t+2 \pi)=p(t)$.

(A3) For all $x \neq 0$,

$$
\gamma x g(x) \geq x^{2} \dot{g}(x)>0, \quad x g(x) \geq(\alpha+1) G(x),
$$

with some constants $0<\gamma, \alpha<1$ and

$$
\left|x^{k} G^{(k)}(x)\right| \leq C \cdot G(x), \quad k \geq 3 .
$$

(A4) For all $x \neq 0$,

$$
\left|x^{k} F^{(k)}(x)\right| \leq C \cdot|g(x)|, \quad k \geq 0 .
$$

Now we are ready to state our main result.

Theorem 1. Assume (A1)-(A4) holds. Then every solution of (1.1) is bounded; that is, if $x=x(t)$ is a solution of $(1.1)$, then it is defined in $(-\infty,+\infty)$ and

$$
\sup _{t \in R}(|x(t)|+|\dot{x}(t)|)<+\infty .
$$


Remark 1. The assumptions on the functions $F$ and $G$ can be weakened to the requirement that they hold for $x \geq d$ for any fixed constant $d>0$.

2. It is enough to assume that $g(x), f(x)$ and $p(t)$ are finitely smooth.

3. From (A2), the functions $F$ and $G$ are even.

4. From (A3), it is easy to see that

$$
(\alpha+1) G(x) \leq x g(x) \leq(\gamma+1) G(x) .
$$

5. The assumptions (A3) and (A4) in some sense are necessary. For example, from [2] we know that the equation

$$
\ddot{x}+(2 m+1)^{2} x+\frac{1}{(2 m+1)^{2}} \arctan x \sin (2 m+1) t
$$

has no periodic solutions. Hence, there is at least one unbounded solution. Also, the example $\ddot{x}+x \dot{x}+x=0$ with the unbounded solution $x(t)=-t$ shows that some condition on $f$ or $F$ is necessary.

6. To explain the role of assumption (A2), we first note that (1.1) is not a Hamiltonian system. This means that the classical twist theorem of Moser is not applicable, and to compensate for this, a possibility is to suppose that (1.1) is reversible, i.e., the assumption (A2) holds. Under these assumptions, the Poincaré map that results after a suitable series of transformations will be reversible as well, and we can try to rely on the KAM theorem for reversible diffeomorphisms that guarantees the existence of invariant curves for such reversible maps and thus the boundedness of all solutions (see Lemma A.3).

The rest of the paper is organized as follows. The proof of Theorem 1 will be given in Section 3. Section 2 deals with some technical lemmas that are employed in the proof of the main result. In the Appendix we recall some basic definitions and properties of reversible systems.

\section{Some transformations AND ESTIMATES}

We first rewrite (1.1) as

$$
\left\{\begin{array}{l}
\dot{x}=\lambda y-F(x) \\
\dot{y}=-\lambda x-\lambda^{-1} g(x)+\lambda^{-1} p(t) .
\end{array}\right.
$$

From assumption (A2) we know that (2.1) is reversible with respect to the transformation $(x, y) \mapsto(-x, y)$; see the Appendix for the details.

Passing to polar coordinates

$$
x=r \sin \theta, \quad y=r \cos \theta,
$$

after a short calculation (2.1) reads as

$$
\left\{\begin{array}{l}
\dot{r}=M_{1}(r, \theta)+N_{1}(r, \theta)+L_{1}(r, \theta, t) \\
\dot{\theta}=\lambda+M_{2}(r, \theta)+N_{2}(r, \theta)+L_{2}(r, \theta, t)
\end{array}\right.
$$

where

$$
\begin{array}{ll}
M_{1}(r, \theta)=-\lambda^{-1} \cos \theta g(r \sin \theta), & M_{2}(r, \theta)=\lambda^{-1} r^{-1} \sin \theta g(r \sin \theta), \\
N_{1}(r, \theta)=-\sin \theta F(r \sin \theta), & N_{2}(r, \theta)=-r^{-1} \cos \theta F(r \sin \theta), \\
L_{1}(r, \theta, t)=\lambda^{-1} p(t) \cos \theta, & L_{2}(r, \theta, t)=-\lambda^{-1} r^{-1} p(t) \sin \theta .
\end{array}
$$

From assumption (A2) we know that $M_{1}, N_{1}$ are odd in $\theta, L_{1}$ is even in $\theta$ and odd in $t$, and $M_{2}, N_{2}$ are even in $\theta, L_{2}$ is odd in $\theta$ and odd in $t$. Therefore, (2.2) is reversible with respect to the transformation $(\theta, r) \mapsto(-\theta, r)$. 
Denote by $J(r)$ the average value of $\lambda^{-2} M_{2}(r, \theta)$ over $S^{1}$, that is

$$
J(r)=\frac{1}{2 \pi} \int_{0}^{2 \pi} \lambda^{-2} M_{2}(r, \theta) d \theta .
$$

Now we will give some estimates on the functions $M_{i}, N_{i}$ and $L_{i}(i=1,2)$.

Lemma 2.1. The following inequalities hold for all nonnegative integers $k$ and $l$ :

$$
\begin{array}{ll}
\left|r^{k} \partial_{r}^{k} M_{1}(r, \theta)\right| \leq C \cdot\left|M_{1}(r, \theta)\right| \leq C \cdot r^{\gamma}, \\
\left|r^{k} \partial_{r}^{k} M_{2}(r, \theta)\right| \leq C \cdot\left|M_{2}(r, \theta)\right| \leq C \cdot r^{\gamma-1}, \\
\left|r^{k} \partial_{r}^{k} N_{1}(r, \theta)\right| \leq C \cdot\left|M_{1}(r, \theta)\right| \leq C \cdot r^{\gamma}, \\
\left|r^{k} \partial_{r}^{k} N_{2}(r, \theta)\right| \leq C \cdot\left|M_{2}(r, \theta)\right| \leq C \cdot r^{\gamma-1}, \\
\left|r^{k} \partial_{r}^{k} \partial_{t}^{l} L_{1}(r, \theta, t)\right| \leq C, \\
\left|r^{k} \partial_{r}^{k} \partial_{t}^{l} L_{2}(r, \theta, t)\right| \leq C \cdot r^{-1} .
\end{array}
$$

Proof. This lemma can be proved easily by the assumption (A3), (A4) and Remark 4.

The following lemma gives some estimates on the function $J(r)$ which can be proved easily.

Lemma 2.2. The following inequalities hold for all nonnegative integers $k$ :

$$
\begin{gathered}
c \cdot r^{\alpha-1} \leq J(r) \leq C \cdot r^{\gamma-1}, \\
r\left|J^{\prime}(r)\right| \geq(1-\gamma) J(r), \quad\left|r^{k} J^{(k)}(r)\right| \leq C \cdot J(r) .
\end{gathered}
$$

Because of $\frac{d \theta}{d t} \neq 0$ for all $r \gg 1$ from Lemma 2.1, (2.2) is equivalent to the system

$$
\left\{\begin{array}{l}
\frac{d r}{d \theta}=M_{0}(r, \theta)+L_{3}(r, t, \theta), \\
\frac{d t}{d \theta}=\lambda^{-1}-\lambda^{-2} M_{2}(r, \theta)-\lambda^{-2} N_{2}(r, \theta)+L_{4}(r, t, \theta),
\end{array}\right.
$$

where

$$
\begin{aligned}
& M_{0}(r, \theta)=\left(M_{1}+N_{1}\right) \cdot\left(\lambda^{-1}-\lambda^{-2} M_{2}-\lambda^{-2} N_{2}\right), \\
& L_{3}(r, t, \theta)=L_{1} \cdot\left(\lambda^{-1}-\lambda^{-2} M_{2}-\lambda^{-2} N_{2}\right)+L_{4} \cdot\left(M_{1}+N_{1}+L_{1}\right), \\
& L_{4}(r, t, \theta)=\left[\lambda+M_{2}+N_{2}+L_{2}\right]^{-1}-\lambda^{-1}+\lambda^{-2} M_{2}+\lambda^{-2} N_{2} .
\end{aligned}
$$

It is easy to see that the function $M_{0}$ is odd in $\theta$ which satisfies the same estimate as $M_{1}$ in Lemma 2.1 and

$$
L_{3}(r,-t,-\theta)=-L_{3}(r, t, \theta), \quad L_{4}(r,-t,-\theta)=L_{4}(r, t, \theta) .
$$

Therefore, this system (2.3) is reversible with respect to the transformation $(t, r) \mapsto$ $(-t, r)$.

It is not difficult to verify that the functions $L_{3}$ and $L_{4}$ satisfy for all nonnegative integers $k$ and $l$,

$$
\left|r^{k} \partial_{r}^{k} \partial_{t}^{l} L_{3}(r, t, \theta)\right| \leq C \cdot \max \left\{\left|L_{1}(r, \theta, t)\right|, r^{-2}\left|M_{1}(r, \theta)\right|^{3}\right\}
$$

and

$$
\left|r^{k} \partial_{r}^{k} \partial_{t}^{l} L_{4}(r, t, \theta)\right| \leq C \cdot \max \left\{\left|L_{2}(r, \theta, t)\right|,\left|M_{2}(r, \theta)\right|^{2}\right\} .
$$

Now we will introduce a series of $\mathcal{G}$-invariant transformations such that in the transformed system, the terms corresponding to the term $M_{0}$ have the same growth speed as the terms $L_{3}$ if the variable $r$ is sufficiently large.

For $r_{0}>0$, we define the domain

$$
A_{r_{0}}=\left\{(r, t, \theta) \mid r \geq r_{0},(t, \theta) \in T^{2}\right\}, \quad T^{2}=S^{1} \times S^{1} .
$$


Lemma 2.3. There exists a $\mathcal{G}$-invariant diffeomorphism $\Psi_{1}$, having the form

$$
\Psi_{1}: r=\rho+U_{1}(\rho, \theta), \quad t=t,
$$

where $U_{1}(\rho, \theta+2 \pi)=U_{1}(\rho, \theta)$ such that $A_{r_{-}} \subset \Psi_{1}\left(A_{r_{0}}\right) \subset A_{r_{+}}$for some large $r_{-}<r_{0}<r_{+}$. Under this transformation, (2.3) is transformed into the system

$$
\left\{\begin{array}{l}
\frac{d \rho}{d \theta}=R_{1}(\rho, t, \theta) \\
\frac{d t}{d \theta}=\lambda^{-1}-\lambda^{-2} M_{2}(\rho, \theta)-\lambda^{-2} N_{2}(\rho, \theta)+R_{2}(\rho, t, \theta)
\end{array}\right.
$$

where the functions $R_{1}$ and $R_{2}$ satisfy the same estimates as $L_{3}$ and $L_{4}$. Moreover, the new system (2.4) is reversible with respect to the transformation $(t, \rho) \mapsto(-t, \rho)$.

Proof. Define a transformation $\Phi_{1}$ by

$$
\Phi_{1}: \rho=r+V_{1}(r, \theta), \quad t=t
$$

where

$$
V_{1}(r, \theta)=-\int_{0}^{\theta} M_{0}(r, s) d s
$$

Since $M_{0}$ is odd in $\theta$ and $\left|r^{k} \partial_{r}^{k} M_{0}(r, \theta)\right| \leq C \cdot r^{\gamma}$, we have

(1) $V_{1}(r, \theta+2 \pi)=V_{1}(r, \theta)$;

(2) $V_{1}(r,-\theta)=V_{1}(r, \theta)$;

(3) the transformation $\Phi_{1}$ is a diffeomorphism and satisfies

$$
\left|r^{k} \partial_{r}^{k} V_{1}(r, \theta)\right| \leq C \cdot\left|M_{1}(r, \theta)\right| \leq C \cdot r^{\gamma} .
$$

Let $\Psi_{1}=\Phi_{1}^{-1}: r=\rho+U_{1}(\rho, \theta), t=t$. Then (2.3) is transformed into

$$
\left\{\begin{array}{l}
\frac{d \rho}{d \theta}=\widetilde{M}_{0}(\rho, \theta)+\widetilde{L}_{3}(\rho, t, \theta), \\
\frac{d t}{d \theta}=\lambda^{-1}-\lambda^{-2} M_{2}(\rho, \theta)-\lambda^{-2} N_{2}(\rho, \theta)+\widetilde{L}_{4}(\rho, t, \theta),
\end{array}\right.
$$

where

$$
\begin{aligned}
& \tilde{M}_{0}(\rho, \theta)=\partial_{r} V_{1}(r, \theta) \cdot M_{0}(r, \theta) \\
& \tilde{L}_{3}(\rho, t, \theta)=L_{3}(r, t, \theta) \cdot\left(1+\partial_{r} V_{1}(r, \theta)\right) \\
& \tilde{L}_{4}(\rho, t, \theta)=L_{4}(r, t, \theta)+\lambda^{-2}\left[M_{2}(\rho, \theta)+N_{2}(\rho, \theta)-M_{2}(r, \theta)-N_{2}(r, \theta)\right]
\end{aligned}
$$

and $r=\rho+U_{1}(\rho, \theta)$.

In order to estimate the new functions in (2.5), we need

Claim. For $\rho$ large enough, we have

(1) $\left|\rho^{k} \partial_{\rho}^{k} U_{1}(\rho, \theta)\right| \leq C \cdot\left|M_{1}(\rho, \theta)\right| \leq C \cdot \rho^{\gamma}$;

(2) $U_{1}(\rho,-\theta)=U_{1}(\rho, \theta)$.

The proof is similar to that of Lemma 5.1 in 7 .

From this claim, it follows that the transformation $\Psi_{1}$ is a $\mathcal{G}$-invariant diffeomorphism, which implies that the transformed system (2.5) is a reversible system with respect to the transformation $\mathcal{G}:(t, \rho) \mapsto(-t, \rho)$. Moreover, $\widetilde{M}_{0}(\rho, \theta)$ is odd in $\theta$. By direct computations, we know that $\widetilde{L}_{3}$ and $\widetilde{L}_{4}$ satisfy the same estimates as those of $L_{3}$ and $L_{4}$, and $\widetilde{M}_{0}(\rho, \theta)$ satisfies

$$
\left|\rho^{k} \partial_{\rho}^{k} \widetilde{M}_{0}(\rho, \theta)\right| \leq C \cdot \rho^{\gamma-1} \cdot\left|M_{0}(\rho, \theta)\right| .
$$

We note that the transformed system (2.5) satisfies all the assumptions of Lemma 2.3. Hence, there is an integer $j$ such that after $j$ successive applications of the above 
step we find that the corresponding term $M_{0}$ satisfies the same estimate as $L_{3}$. At the same time, we rewrite the system as

$$
\left\{\begin{array}{l}
\frac{d \rho}{d \theta}=R_{1}(\rho, t, \theta) \\
\frac{d t}{d \theta}=\lambda^{-1}-\lambda^{-2} M_{2}(\rho, \theta)-\lambda^{-2} N_{2}(\rho, \theta)+R_{2}(\rho, t, \theta)
\end{array}\right.
$$

where the functions $R_{1}$ and $R_{2}$ satisfy the same estimates as $L_{3}$ and $L_{4}$. The proof of Lemma 2.3 is finished.

Now we will introduce a $\mathcal{G}$-invariant transformation such that in the transformed system, the terms corresponding to the terms $M_{2}$ and $N_{2}$ do not depend on the variable $\theta$.

Lemma 2.4. There exists a $\mathcal{G}$-invariant diffeomorphism $\Psi_{2}$, having the form

$$
\Psi_{2}: \rho=\rho, \quad t=\tau+U_{2}(\rho, \theta),
$$

where $U_{2}(\rho, \theta+2 \pi)=U_{2}(\rho, \theta)$ such that $A_{\rho_{-}} \subset \Psi_{2}\left(A_{\rho_{0}}\right) \subset A_{\rho_{+}}$for some large $\rho_{-}<\rho_{0}<\rho_{+}$. Under this transformation, (2.6) is transformed into the system

$$
\left\{\begin{array}{l}
\frac{d \rho}{d \theta}=Q_{1}(\rho, \tau, \theta), \\
\frac{d \tau}{d \theta}=\lambda^{-1}+J(r)+Q_{2}(\rho, \tau, \theta),
\end{array}\right.
$$

where the functions $Q_{1}$ and $Q_{2}$ satisfy the same estimates as $R_{1}$ and $R_{2}$. Moreover, the new system (2.7) is reversible with respect to the transformation $(\tau, \rho) \mapsto$ $(-\tau, \rho)$.

Proof. The proof is very similar to the given one for Lemma 2.3 , and we omit it here.

\section{The Proof of Theorem 1}

From the discussions in $\S 2$, it follows that (1.1) is transformed into the system

$$
\left\{\begin{array}{l}
\frac{d \rho}{d \theta}=Q_{1}(\rho, \tau, \theta), \\
\frac{d \tau}{d \theta}=\lambda^{-1}-J(\rho)+Q_{2}(\rho, \tau, \theta) .
\end{array}\right.
$$

In order to apply the small twist theorem for reversible diffeomorphims, we introduce a new variable $\nu$ varying in the closed interval $[1,2]$ and a small positive parameter $\varepsilon$ by the formula

$$
J(\rho)=\varepsilon \nu .
$$

From Lemma 2.2 it follows that

$$
\rho \gg 1 \Longleftrightarrow \varepsilon \ll 1 .
$$

Now (3.1) is equivalent to the system

$$
\left\{\begin{array}{l}
\frac{d \nu}{d \theta}=W_{1}(\nu, \tau, \theta, \varepsilon), \\
\frac{d \tau}{d \theta}=\lambda^{-1}-\varepsilon \nu+W_{2}(\nu, \tau, \theta, \varepsilon),
\end{array}\right.
$$

where

$$
W_{1}(\nu, \tau, \theta, \varepsilon)=\varepsilon^{-1} J^{\prime}(\rho) Q_{1}(\rho(\varepsilon \nu), \tau, \theta), \quad W_{2}(\nu, \tau, \theta, \varepsilon)=Q_{2}(\rho(\varepsilon \nu), \tau \theta),
$$

and $\rho=\rho(\varepsilon \nu)$ is defined implicitly by $(3.2)$.

Now we give the estimates on the functions $W_{1}$ and $W_{2}$. 
Lemma 3.1. The functions $W_{1}$ and $W_{2}$ possess the following estimates for all nonnegative integers $k$ and $l$ :

$$
\left|\partial_{\nu}^{k} \partial_{t}^{l} W_{i}\right| \leq C \cdot \varepsilon^{1+\sigma}, \quad i=1,2
$$

where

$$
\sigma:=\min \left\{1, \frac{\alpha}{1-\alpha}\right\}>0
$$

Proof. First, we estimate $W_{2}$. By the estimate on $Q_{2}$, we have

$$
\left|W_{2}\right|=\left|Q_{2}(\rho(\varepsilon \nu), \tau, \theta)\right| \leq C \cdot \max \left\{\rho^{-1}, J(\rho)^{2}\right\} \leq C \cdot \varepsilon^{1+\sigma} .
$$

We see that $\partial_{\tau}^{l} \partial_{\nu}^{k} Q_{2}(\rho(\varepsilon \nu), \tau, \theta)$ is a sum of terms of the form

$$
\partial_{\tau}^{l} \partial_{\rho}^{s} Q_{2}(\rho(\varepsilon \nu)) \cdot \partial_{\nu}^{k_{1}} \rho(\varepsilon \nu) \cdots \partial_{\nu}^{k_{s}} \rho(\varepsilon \nu)
$$

where $k_{1}+\cdots+k_{s}=k, 1 \leq s \leq k$. It is easy to verify that

$$
\left|\partial_{\nu}^{k} \rho(\varepsilon \nu)\right| \leq C \cdot \rho .
$$

So we obtain

$$
\left|\partial_{\tau}^{l} \partial_{\nu}^{k} W_{2}(\nu, \tau, \theta, \varepsilon)\right| \leq \max \left\{\rho^{-1}, J(\rho)^{2}\right\} \leq C \cdot \varepsilon^{1+\sigma} .
$$

Second, we estimate $W_{1}$. We have

$$
\left|W_{1}\right|=\left|\varepsilon^{-1} J^{\prime}(\rho) Q_{1}(\rho(\varepsilon \nu), \tau, \theta)\right| \leq C \cdot \max \left\{\rho^{-1}, J(\rho)^{3}\right\} \leq C \cdot \varepsilon^{1+\sigma} .
$$

Similar to the estimates on $W_{1}$, it is easy to verify that the following inequalities hold for all nonnegative integers $k$ and $l$ :

$$
\left|\partial_{\tau}^{l} \partial_{\nu}^{k} W_{1}\right| \leq C \cdot \varepsilon^{1+\sigma} .
$$

Now we are in a position to prove Theorem 1 stated in the introduction.

Proof of Theorem 1. Since the functions $W_{1}$ and $W_{2}$ satisfy the estimates in Lemma 3.1 , one can verify easily that the solutions of (3.3) do exist for $0 \leq \theta \leq 2 \pi$ if the parameter $\varepsilon$ is sufficiently small. Integrating (3.3) from $\theta=0$ to $\theta=2 \pi$ we obtain the Poincaré map $P$ of the form

$$
\tau_{1}=\tau_{0}+2 \pi \lambda-2 \pi \varepsilon \nu_{0}+\Xi_{1}\left(\tau_{0}, \nu_{0}, \varepsilon\right), \quad \nu_{1}=\nu_{0}+\Xi_{2}\left(\tau_{0}, \nu_{0}, \varepsilon\right)
$$

with $\Xi_{1,2}$ still satisfying the same estimates as $W_{1,2}$. Moreover, it is reversible with respect to $\mathcal{G}:\left(\tau_{0}, \nu_{0}\right) \mapsto\left(-\tau_{0}, \nu_{0}\right)$. Therefore, the Poincaré map $P$ meets all the conditions of Lemma A.3, and it possesses a sequence of invariant curves tending to infinity. Hence, in the original system (1.1), there is a corresponding sequence of invariant tori in phase space $(t, x, \dot{x}) \in S^{1} \times R^{2}$. Moreover, those invariant tori has positive Lebesgue measure, and the union of its interior is $R^{2}$. Since any solution of system (1.1) must stay within one of those tori, it is bounded. The proof is thus finished. 


\section{ApPendix: Reversible SYSTEMS}

We recall some definitions and facts related to reversible systems; see [20] for more information.

Definition A.1. Let $X: \Omega \times R \rightarrow R^{n}$ be continuous and 1-periodic in the last variable, with $\Omega \subset R^{n}$ an open set. The system

$$
\dot{x}=X(x, t)
$$

is called a reversible system if there is an involution $\mathcal{G}: R^{n} \rightarrow R^{n}$ (that is, $\mathcal{G}$ is a $C^{1}$-diffeomorphism and $\left.\mathcal{G}^{2}=I_{R^{n}}\right)$, with $\mathcal{G}(\Omega)=\Omega$ and such that

$$
D \mathcal{G}(\mathcal{G} x) \cdot X(\mathcal{G} x,-t)=-X(x, t), \quad \forall(x, t) \in \Omega \times R .
$$

(Here and thereafter, $D \mathcal{G}$ stands for the Jacobian matrix of $\mathcal{G}$.)

Similarly, we give a definition of reversible diffeomorphisms, as follows.

Definition A.2. Let $A: R^{n} \supset \Omega \rightarrow R^{n}$ be a homeomorphism onto its image, and let $\mathcal{G}: R^{n} \rightarrow R^{n}$ be a homeomorphism with $\mathcal{G}^{2}=I_{R^{n}}$. We say that $A$ is reversible with respect to $\mathcal{G}$ on a set $\mathcal{D} \subset \Omega \cap A(\Omega)$, with $\mathcal{G}(\mathcal{D})=\mathcal{D}$, if

$$
A^{-1}=\mathcal{G} A \mathcal{G}, \quad \forall x \in \mathcal{D}
$$

holds.

The next result (which is almost obvious from the definitions) is very useful for our applications.

Lemma A.1. Assume uniqeness of the solutions for the Cauchy problems associated to (A.1), and let the time 1-map $\varphi^{1}$ and its inverse $\left(\varphi^{1}\right)^{-1}$ be defined. Then $\varphi^{1}$ is a reversible homeomorphism with respect to an involution $\mathcal{G}: R^{n} \rightarrow R^{n}$ provided that (A.1) is reversible with respect to $\mathcal{G}$.

The next definition is useful when changes of variables are involved.

Definition A.3. Assume that $T(\cdot, t)$ is an invertible transformation of $\Omega$ for any fixed $t$ and that $\mathcal{G}$ is an involution of $R^{n}$ with $\mathcal{G}(\Omega) \Omega$. We say that $T(x, t)$ is $\mathcal{G}$-invariant if the equality

$$
\mathcal{G} \circ T(x, t)=T(\mathcal{G} x,-t)
$$

holds.

Lemma A.2. Suppose that the system (A.1) is reversible with respect to an involution $\mathcal{G}: R^{n} \rightarrow R^{n}$. If a transformation $T(\cdot, t): \Omega \rightarrow R^{n}$ is $\mathcal{G}$-invariant and $C^{1}$ in $x$ and $t$, then the transformed system of (A.1) under $T$ is also reversible with respect to $\mathcal{G}$.

Now we give the KAM theorem of reversible diffeomorphisms as a lemma.

Lemma A.3 ([20]). Let $\Omega \subset R^{m}$ be a closed ball of radius 1 and $D_{*} \subset C^{m}$ a complex neighborhood of $\Omega$. Let $r_{0}, \tilde{r}_{0} \in(0,1]$. Denote by $D$ the following domain in $C^{m}$ :

$$
D=\left\{x=\left(x_{1}, \ldots, x_{m}\right) \in C^{m}:\left|\operatorname{Im} x_{j}\right|<r_{0}, 1 \leq j \leq m\right\} \times\left\{y \in C^{m}: y \in D_{*}\right\} .
$$

Suppose that $\gamma, c \in(0,1]$ are fixed and the following mappings are given on $D$ :

$$
A:(x, y) \mapsto\left(x+\gamma y+f^{1}(x, y), y+f^{2}(x, y)\right)
$$


and

$$
\mathcal{G}:(x, y) \mapsto(-x, y),
$$

where $f^{1}$ and $f^{2}$ are normal in $D$; that is, $f^{1}$ and $f^{2}$ are holomorphic, $2 \pi$-periodic in $x$ and real-valued on $D \cap R^{2 m}$.

Assume that $A^{-1}=\mathcal{G} A \mathcal{G}$ on $D$. Introduce the notation

$$
\Omega_{\gamma, c}=\left\{\omega \in \gamma \Omega:\left|\frac{(q, \omega)}{2 \pi}-p\right| \geq \frac{\gamma c}{|q|^{m+1}}\right\}
$$

for all $q \in Z^{m} /\{0\}$ and $p \in Z$.

Then for each $\epsilon>0$, there is a $\delta>0$, depending only on $\epsilon, D$ and $c$ but not on $\gamma$, such that if $f^{1}, f^{2}<\gamma \delta$ on $D$, then for each $\omega \in \Omega_{\gamma, c}$, the mappings $A$ and $\mathcal{G}$ have a common invariant $m$-dimensional manifold

$$
x=\phi+\Phi_{\omega}^{1}(\phi), \quad y=\gamma^{-1} \omega+\Phi_{\omega}^{2}(\phi),
$$

where $\Phi^{1}$ and $\Phi^{2}$ are normal in the domain

$$
\left\{\phi=\left(\phi_{1}, \ldots, \phi_{m}\right) \in C^{m}:\left|\operatorname{Im} \phi_{j}\right|<\frac{r_{0}}{2}, 1 \leq j \leq m\right\},
$$

such that the diffeomorphisms of the manifold (A.2) induced by the mappings $A$ and $\mathcal{G}$ are $\phi \mapsto \phi+\omega$ and $\phi \mapsto-\phi$, respectively. Moreover, the following inequality holds:

$$
\left|\Phi_{\omega}^{1}\right|,\left|\Phi_{\omega}^{2}\right|<\epsilon .
$$

Remark. The proof which can be found in 20] is very similar to the one for Moser's twist theorem, and the intersection property assumption is replaced by the reversibility assumption here. So in the case of $C^{\infty}$-perturbations or finite smooth perturbations, the statement of the above lemma is still true (see [14]).

\section{ACKNOWLEDGMENT}

The author is very grateful to Professors Tongren Ding, Bin Liu and Meirong Zhang for their valuable suggestions. Also, the author is indebted to the referee for his careful reading of the manuscript and suggestions.

\section{REFERENCES}

[1] R. Dieckerhoff and E. Zehnder, Boundedness of solutions via the twist theorem, Ann. Scuola Norm. Sup. Pisa Cl. Sci., 14 (1987), 79-95. MR 89e:34066

[2] T. Ding, Nonlinear oscillations at a point of resonance, Scientia Sinica Ser. A, 25 (1982), 918-931. MR 84c:34058

[3] M. Kunze, T. Kupper and B. Liu, Boundedness and unboundedness of solutions for reversible oscillators at resonance, Nonlinearity, 14(5) (2001), 1105-1122. MR 2002g:34079

[4] T. Kupper and J. You, Existence of quasiperiodic solutions and Littlewood's boundedness problem of Duffing equations with subquadratic potentials, Nonlinear Anal., 35 (1999), 549559. MR 99i:34064

[5] M. Levi, Quasi-periodic motions in superquadratic time-periodic potentials, Comm. Math. Phys., 143(1) (1991), 43-83. MR 93i:34080

[6] X. Li, Boundedness of solutions for sublinear reversible systems, Science in China (Series A), 44(2) (2001), 137-144. MR 2002a:34054

[7] X. Li, Boundedness of solutions for superlinear reversible systems, Chinese Ann. Math. (Series B), 22B(1) (2001), 31-46. MR 2002a:34053

[8] X. Li, Invariant tori for semilinear reversible systems, preprint.

[9] B. Liu, On Littlewood's boundedness problem for sublinear Duffing equations, Trans. Amer. Math. Soc., 353(4) (2001), 1567-1585. MR 2001m:34084 
[10] B. Liu, Boundedness in asymmetric oscillations, J. Math. Anal. Appl., 231 (1999), 355-373. MR 2000c:34093

[11] B. Liu, Boundedness in nonlinear oscillations at resonance, J. Differential Equations, 153 (1999), 142-174. MR 2000d:34075

[12] B. Liu, Boundedness of solutions for semilinear Duffing equations, J. Differential Equations, 145 (1998), 119-144. MR 99e:34041

[13] B. Liu and F. Zanolin, Boundedness of solutions for second order quasilinear ODEs, preprint.

[14] B. Liu, Invariant curves of reversible mappings with small twist, preprint.

[15] B. Liu, An application of KAM theorem of reversible systems, Sci. China Ser. A, 34 (1991), 1068-1078. MR 93d:58143

[16] G. Morris, A case of boundedness in Littlewood's problem on oscillatory differential equations, Bull. Austral. Math. Soc., 14 (1976), 71-93. MR 53:6019

[17] R. Ortega, Invariant curves of mappings with averaged small twist, Advanced Nonlinear Studies, 1 (2001), 14-39. MR 2002h:37067

[18] R. Ortega, Boundedness in a piecewise linear oscillator and a variant of the small twist theorem, Proceedings London Math. Soc., 79 (1999), 381-413. MR 2000g:34055

[19] R. Ortega, Asymmetric oscillators and twist mappings, J. London Math. Soc., 53 (1996), 325-342. MR 96k:34093

[20] M. Sevryuk, Reversible systems, Lecture Notes in Mathematics, Vol. 1211, Springer-Verlag, Berlin, 1986. MR 88b:58058

[21] J. You, Boundedness for solutions of superlinear Duffing equations via the twist theorem, Sci. China Ser. A, 35 (1992), 399-412. MR 95c:34067

[22] R. Yuan, Quasiperiodic solutions and boundedness of solutions for a class of nonlinear differential equations of second order, Nonlinear Anal., 31 (1998), 649-664. MR 99a:34130

[23] X. Yuan, Invariant tori of Duffing-type equations, J. Differential Equations, 142 (1998), 231-262. MR 99a:34135

Department of Mathematics, Beijing Normal University, Beijing 100875, People's Republic of China

E-mail address: xli@bnu.edu.cn 